Document downloaded from:

http://hdl.handle.net/10251/106424

This paper must be cited as:

Barbi, M.; Pérez Simbor, S.; García Pardo, C.; Andreu Estellés, C.; Cardona Marcet, N. (2018). Localization for capsule endoscopy at UWB frequencies using an experimental multilayer phantom. Institute of Electrical and Electronics Engineers (IEEE). doi:10.1109/WCNCW.2018.8369015

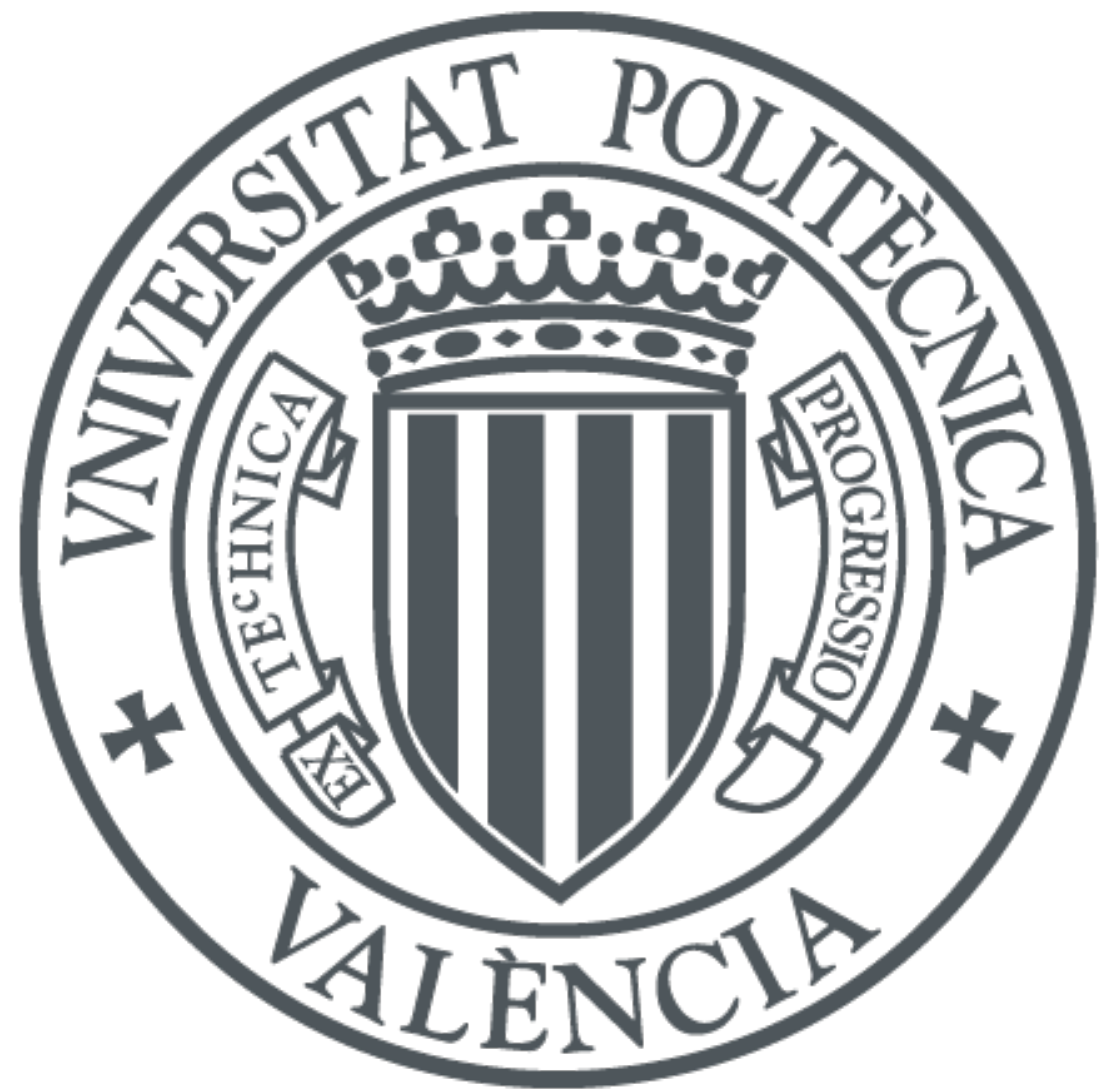

The final publication is available at

https://doi.org/10.1109/WCNCW.2018.8369015

Copyright Institute of Electrical and Electronics Engineers (IEEE)

Additional Information 


\title{
Localization for Capsule Endoscopy at UWB Frequencies using an Experimental Multilayer Phantom
}

\author{
Martina Barbi, Sofia Perez-Simbor, Concepcion Garcia-Pardo, \\ Carlos Andreu, Narcís Cardona \\ Institute of Telecommunications and Multimedia Applications (ITeAM) \\ Universitat Politècnica de València \\ 46022, Valencia, Spain \\ marbar6@iteam.upv.es
}

\begin{abstract}
Localization inside the human body using ultrawideband (UWB) wireless technology is gaining importance in several medical applications such as capsule endoscopy. Performance analysis of RF based localization techniques are mainly conducted through simulations using numerical human models or through experimental measurements using homogeneous phantoms. One of the most common implemented RF localization approaches uses the received signal strength (RSS). However, to the best of our knowledge, no experimental measurements employing multilayer phantoms are currently available in literature. This paper investigates the performance of RSS-based technique for two-dimensional (2D) localization by employing a two-layer experimental phantom-based setup. Preliminary results on the estimation of the in-body antenna coordinates show that RSS-based method can achieve a location accuracy on average of $0.5-1 \mathrm{~cm}$ within a certain range of distances between in-body and on-body antenna.
\end{abstract}

Index Terms-Wireless capsule endoscopy (WCE), UltraWideband (UWB), Received Signal Strength (RSS), heterogeneous phantom.

\section{INTRODUCTION}

Wireless Capsule Endoscopy (WCE) is a remarkable and attractive technology adopted in the biomedical sector. It provides a non-invasive wireless imaging technology for the entire gastrointestinal (GI) tract. WCE allows specialists to recognize and diagnose diseases affecting the whole GI tract. Although physicians can receive clear pictures of abnormalities in the GI tract, they have no information about their exact location. Precise localization of the detected disorders is crucial for the subsequent removal procedure by surgery. Thus, the potential of WCE in replacing the conventional gastrointestinal endoscopy and colonoscopy highly relies on the location and tracking accuracy of the capsule along the GI tract.

In recent years, different approaches for WCE localization have been investigated. These technologies can be distinguished into those employing magnetic field [1], [2], those using imaging algorithms [3], [4], [5], and those based on Radio Frequency (RF) signals [6], [7]. Employing the RF signal used for image transmission to also locate the WCE constitutes an efficient and easy solution that does not increase the complexity of the capsule.

Classical localization approaches based on RF signal consist, as a first step, in the estimation of one or more locationdependent parameters, such as time of arrival (ToA) [8], [9], time difference of arrival (TDoA) [9], received signal strength (RSS) [8], or phase difference of arrival (PDoA) [10], [11]. Then, in a second step, the collected parameters are used to estimate the capsule's position through trilateration method.

One of the most common implemented localization approaches uses the received signal strength due to its simplicity [8], [12]. In this technique the attenuation of the received signal strength can be modeled as a function of the distance from the in-body source. Thus, the accuracy of the path loss model of the propagation channel heavily affects the position estimation.

Currently, the frequency band allocated for capsule endoscopy applications is the MICS band (402-405 MHz). This band offers data rate up to $500 \mathrm{kbps}$, which is insufficient to transmit high quality images. Recently, Ultra-wideband (UWB) technology has been attracting attention as potential candidate for next-generation WCE systems [13]. The advantages of UWB include simple transceiver architectures enabling lowpower consumption, low interference to other systems and wide bandwidth resulting in communications at higher data rate. In addition, UWB systems are capable of high accuracy ranging in challenging multipath environments such as the human body.

In current literature, the majority of studies on RF localization techniques at UWB frequencies is based on Finite Difference Time Domain (FDTD) [12], [14] simulations by employing digital models of the gastrointestinal (GI) tract. Measurement campaigns using homogeneous phantom have also been conducted [15]. Homogeneous phantoms poorly approximate the complexity of the human body as they can mimic only one human tissue. To the best of our knowledge no analysis based on experimental measurements using multilayer phantoms have been reported yet, due to the complexity to 
precisely emulate the electromagnetic properties of different tissues in the entire UWB frequency band. Nevertheless, recent researches carried out at Universitat Politècnica de València (UPV) [16], [17] have accomplished with this necessity. Using chemical solutions, such phantoms emulate with a high accuracy different human body tissues from $0.5 \mathrm{GHz}$ to $18 \mathrm{GHz}$, which includes the entire UWB frequency band.

In this paper, the performance of RSS-based technique for UWB in-body to on-body (IB2OB) communications employing a customized measurement setup, including a two-layer phantom model, are presented. Section II presents the experimental phantom-based setup used for the in-body measurements and describes the measurements methodology. Section III.A illustrates the implementation of RSS-based localization approach for the two-dimensional (2D) case. Performance metrics and obtained results are presented in Section III.B. Finally, conclusions and future research plans are discussed in Section IV.

\section{Measurement SetuP AND Methodology}

For the sake of brevity a brief summary of the implemented phantom-based measurements setup (Figure 1) is given here. A more detailed description of the experimental testbed can be found in [18]. The primary components of the measurement setup are: an anechoic chamber, a Vector Network Analyzer (VNA), a three-dimensional automatic positioner, a two-layer plastic phantom container and a magnetic tracker. All the equipment are software-driven by a laptop.

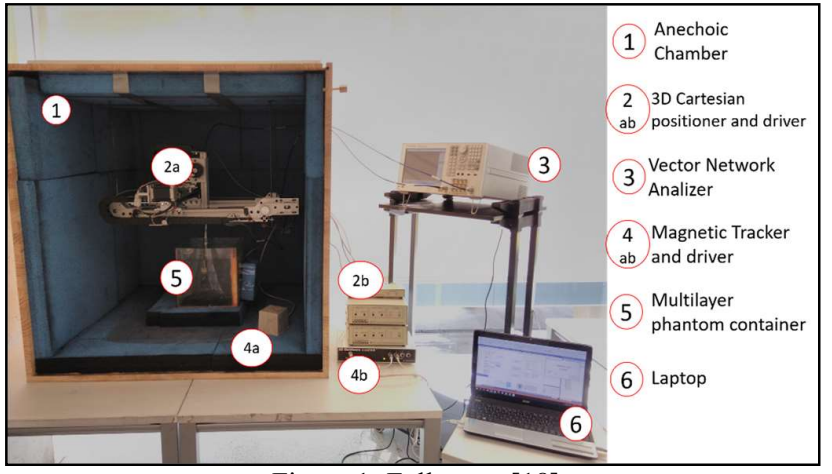

Figure 1: Full setup [18]

The anechoic chamber is used to isolate the interior from the external environment so that undesired contributions from the surroundings will not affect the measurements. The 3D Cartesian positioner (Figure 1, element 2a) is in charge of accurately moving the in-body antenna along the $x, y, z$ axis, inside the liquid phantom. The $3 \mathrm{D}$ magnetic tracker transmitter (Figure 1, element 4a) is the absolute reference system for our localization purposes. Through the generation of a magnetic field and the sensors attached to the in-body and on-body antenna, the distance between antennas as well as the $x, y, z$ coordinates of each antenna can be precisely evaluated.
In WCE scenario colon, muscle and fat are the primary tissues involved in the procedure. Due to the fact that muscle and colon have comparable permittivity, only muscle tissue was employed for the experimental measurements. Muscle-like tissue is the most widely used for this kind of measurements, and, in addition, the muscle phantom created at the UPV [17] is the most accurate so far, covering the whole UWB frequency band. Therefore, the novel phantom container (Figure 1, element 5) was designed for two layers: one for the muscle phantom of dimensions $23 \times 25 \times 25 \mathrm{~cm}^{3}$ and one for the fat phantom of dimensions $2 \times 25 \times 25 \mathrm{~cm}^{3}$.

Lastly, both antennas used are UWB patch antennas [19], [20] with quasi-omnidirectional radiation pattern and dimensions of $2.3 \mathrm{~cm} \times 2 \mathrm{~cm}$ and $5 \mathrm{~cm} \times 4.4 \mathrm{~cm}$ for the in- and on-body antenna, respectively.

During the experimental campaign, the phantom container was located inside the anechoic chamber. The in-body antenna was attached to the 3D Cartesian positioner and immersed in the muscle layer. Such antenna was moved in steps of $1 \mathrm{~cm}$ along $x, y, z$ axis with a grid size of $\left(N_{x}=12, N_{y}=11, N z=2\right)$ as shown in Figure 2a).

Five different positions, with a separation of $2 \mathrm{~cm}$, over the outer fat-like phantom edge (Figure 2a)) were considered for the on-body antenna. For each location, the in-body antenna movement along $x, y, z$ axis was repeated in order to emulate five on-body antennas receiving at the same time. For each inbody to on-body antenna position the forward transmission coefficient $\left(\mathrm{S}_{21}\right)$ was measured in the $3.1-8.5 \mathrm{GHz}$ UWB band, considering 3201 resolution points in frequency. The noise level was assessed to be $-90 \mathrm{dBm}$. For each location, measurements were repeated five times (snapshots) with the aim of improving the Signal-to-Noise Ratio (SNR). Then the average $\mathrm{S}_{21}$ was evaluated.

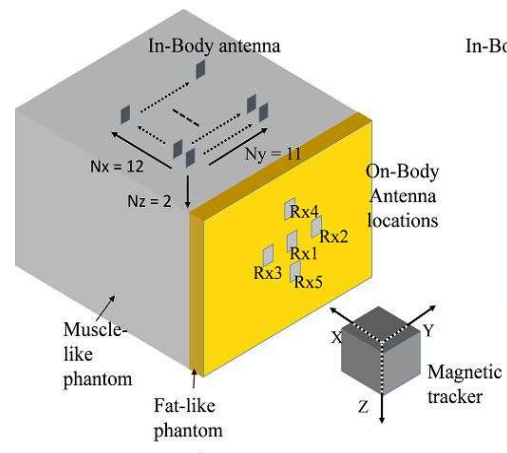

a)

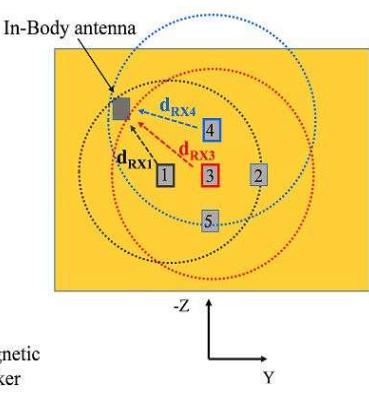

b)
Figure 2. Measured Grid Setup a) and example of 2D localization using a combination of three receivers $b$ )

As the magnetic sensors attached to the antennas are highly sensitive to small movements, for each snapshot, the position 
was calculated 100 times in order to minimize the errors. Then, the distance between antennas as well as their $x, y, z$ coordinates were calculated by averaging the 500 collected measurements, per in-body to on-body position.

\section{ReCEIVED Signal StREnGth (RSS) Positioning}

\section{A. Localization Algorithm}

For the sake of this work, only measurements whose relative received power is above the noise level are taken into account. Specifically, measurements from $3.1-4.1 \mathrm{GHz}$ are considered here.

From the measured $S_{21}$, path loss values for each in-body to on-body antenna position are calculated as follows:

$$
P L(d B)=-10 \log _{10}\left(\operatorname{mean}\left(|H(f)|^{2}\right)\right)
$$

where $\mathrm{H}(\mathrm{f})$ is the frequency transfer function in $N$ resolution points computed as $H(f)=\left|S_{21}\right| e^{-j \phi s_{21}}$, being $\left|S_{21}\right|$ and $\phi_{S_{21}}$ module and phase in radians of the $S_{21}$, respectively.

Considering all the on-body antenna positions (Figure 2a)), path loss values within a distance between in-body and on-body antenna centers $d=4 \mathrm{~cm}$ to $d=8 \mathrm{~cm}$ are fitted by a log-distance approximation model:

$$
P L_{l o g}(d B)=P L_{0, d_{\text {ref }}}(d B)+10 n \log _{10}\left(\frac{d}{d_{\text {ref }}}\right)
$$

where $d$ is the distance between antenna centers, $d_{r e f}$ is the reference distance at $1 \mathrm{~cm}, P L_{0, d_{r e f}}$ is the path loss at $d_{r e f}$ and $n$ is the path loss exponent.

In order to evaluate the performance of the RSS-based technique, a perfect knowledge of the channel is assumed. This means that for each IB2OB antenna position the channel impulse response is assumed to be completely known, i.e., we can detect all the multipath components and therefore, we can precisely compute the path loss at the receiving side. We considered the most ideal case. Through the log-distance fitting model previously evaluated an estimation of $d$ can be obtained as:

$$
d_{\text {est }}=10 \frac{P L_{\text {meas }}-P L_{0, d_{\text {ref }}}}{10 n} \cdot d_{\text {ref }}
$$

where $P L_{\text {meas }}$ is the path loss obtained from measurements using Eqn. (1).

Considering the receivers configuration in Figure 2a), it is important to observe that all receivers are located in the same $(y-z)$ plane i.e., they share the same $x$ coordinate. This results in the possibility to evaluate only two coordinates of the in-body antenna, i.e., $y$ and $z$, as depicted in Figure $2 b$ ).
For the estimation of the in-body antenna coordinates the technique described in [21] is used. The method is suitable for 3D localization but it can be easily adapted for 2D localization. Such methodology is explained in the following.

Let $\mathrm{C}=\left(y_{I B}, z_{I B}\right)$ be the coordinates of the in-body antenna at time instant $\mathrm{t}$ and $R_{i}=\left(y_{i}, z_{i}\right)$ the position of the $i$ th receiver. For $2 \mathrm{D}$ localization, as shown in Figure 2b), at least three receivers are needed, one taken as reference, to get a linear system in 2 unknowns $y_{I B}, z_{I B}$ in the form of:

$$
A x=b
$$

where $A$ and $x$ are given by

$$
\begin{gathered}
A=\left(\begin{array}{cc}
y_{1}-y_{n} & z_{1}-z_{n} \\
\vdots & \vdots \\
y_{N}-y_{n} & z_{N}-z_{n}
\end{array}\right) \\
x=\left(\begin{array}{c}
y_{I B}-y_{n} \\
z_{I B}-z_{n}
\end{array}\right)
\end{gathered}
$$

and $b$ is given by

$$
b=\left(\begin{array}{c}
0.5\left(\hat{d}_{n}(C)^{2}+d_{1 n}{ }^{2}-\hat{d}_{1}(C)^{2}\right) \\
\vdots \\
0.5\left(\hat{d}_{n}(C)^{2}+d_{N n}{ }^{2}-\hat{d}_{N}(C)^{2}\right)
\end{array}\right)
$$

where $R_{n}=\left(y_{n}, z_{n}\right)$ is the position of the reference receiver, $\hat{d}_{n}(C)$ is the estimated distance between the in-body antenna and the $n$th reference receiver, $d_{i n}$ is the distance among the $i$ th receiver and the $n$th reference receiver and $\hat{d}_{i}(C)$ is the estimated distance among the in-body antenna and the $i$ th receiver.

Finally, $\left(y_{I B}, z_{I B}\right)$ are obtained by solving Eqn. (4) as follows:

$$
x=\left(A^{T} A\right)^{-1} A^{T} b
$$

where $(.)^{T}$ represents the transpose of a matrix.

Further details regarding the methodology can be found in [21].

\section{B. Performance Metrics \& Results}

As explained in section $\mathrm{A}$, from the measured $S_{21}$ a $\log$ distance path loss model is obtained for distances among antennas between 4 and $8 \mathrm{~cm}$. Figure 3 depicts the path loss values obtained from measurements along with the related logarithmic fitting (2) being $d_{0}=1 \mathrm{~cm}, P L_{0, \text { dref }}=-26.676 \mathrm{~dB}$ and $n=9.6518$.

From the 2D coordinates the localization error (LE) and its related relative error can be defined as: 


$$
\begin{gathered}
L E=\sqrt{\left(y_{I B}-y_{I B_{-} e s t}\right)^{2}+\left(z_{I B}-z_{I B_{-} e s t}\right)^{2}} \\
\operatorname{RelErr}_{L E}=\frac{\sqrt{\left(y_{I B}-y_{I B_{-} e s t}\right)^{2}+\left(z_{I B}-z_{I B_{-} e s t}\right)^{2}}}{\sqrt{{y_{I B}{ }^{2}+z_{I B}^{2}}^{2}}}
\end{gathered}
$$

where $y_{I B}, z_{I B}$ are the true $(y, z)$ coordinates of the in-body antenna and $y_{I B_{-} e s t}, z_{I B_{-} e s t}$ are the $(y, z)$ coordinates estimated solving Eqn. (8). Furthermore, the relative errors on the estimation of $y_{I B}$ and $z_{I B}$, respectively, can be expressed as:

$$
\begin{aligned}
& \operatorname{RelErr}_{Y_{\text {Yest }}}=\frac{y_{I B_{-} e s t}-y_{I B}}{y_{I B}} \\
& \operatorname{RelErr}_{\mathrm{Z}_{\text {est }}}=\frac{z_{I B_{-} e s t}-z_{I B}}{z_{I B}}
\end{aligned}
$$

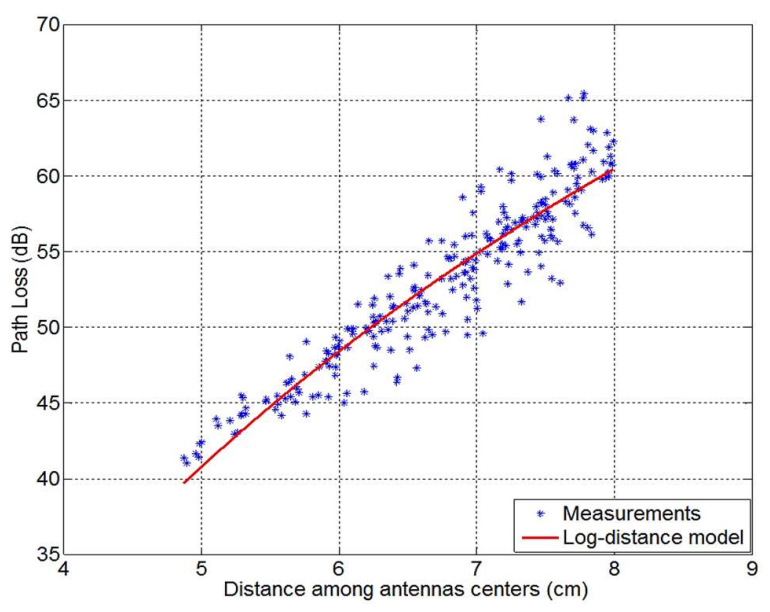

Figure 3. Measured Path Loss and related fitting model

In order to locate the in-body antenna different combinations of three receivers (one of them taken as reference) were used. In this way, the solution of Eqn. (4) will be unique. Here, we accomplished a first estimation of the in-body antenna coordinates by directly solving Eqn. (4) with two equations (2D case).

Figure 4 depicts in a) the relative error on the estimation of the actual in-body antenna location computed as in (10) for several combinations of different receivers, while in b) the Cumulative Distribution Function (CDF) of such relative error is shown. One can observe how the combination of receivers 2, 3 (reference), 4 (red line) performs better compared to the other ones leading to lower relative errors in the position estimation. This is more noticeable in Figure 5 which shows the true inbody antenna locations vs the estimated ones with the three different combinations of receivers. In fact, blue and magenta dots, compared to the red ones, are the most spread out with respect to the true location of the in-body antenna (black dots).
This is due to the fact that receiver 3 (Figure 2a)) is the closest to the first position of movement along $y$ of the in-body antenna, per $\mathrm{cm}$ increase on $x$. Taking it as reference and using receivers 2 and 4, which are located in the direction of movement of the in-body antenna, results in a more accurate estimation compared to the other combinations of receivers.
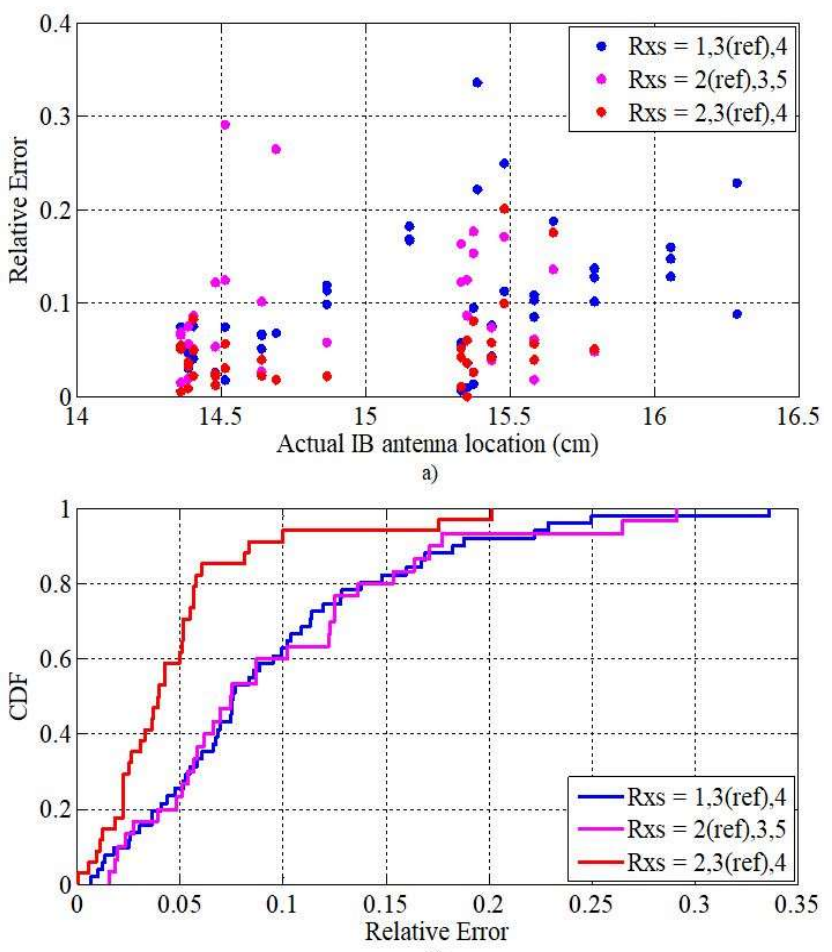

b)

Figure 4. Localization error VS true location of in-body antenna a) and CDF of relative error $b$ )

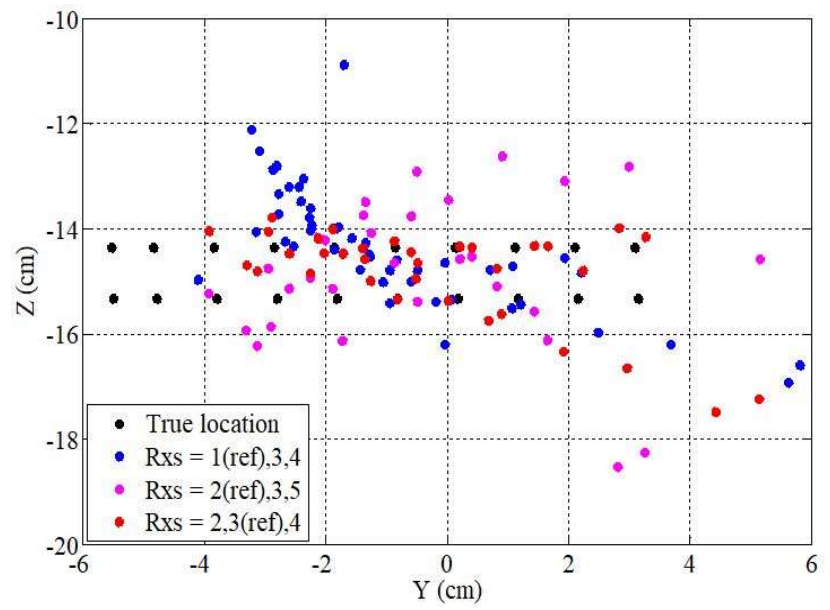

Figure 5. True location of in-body antenna VS estimated location

Figure 6 depicts in a), b) the relative error on the estimation of the in-body antenna coordinates $\left(y_{I B}, z_{I B}\right)$, respectively. One observation is that for all the receivers combinations the relative error on the estimation of $z_{I B}$ is lower than the one for $y_{I B}$. This can be explained looking at Figure 7 where the absolute errors 
on the estimation of $y_{I B}$ (Figure 7a) and on the estimation of $z_{I B}$ (Figure $7 \mathrm{~b}$ ) are reported.
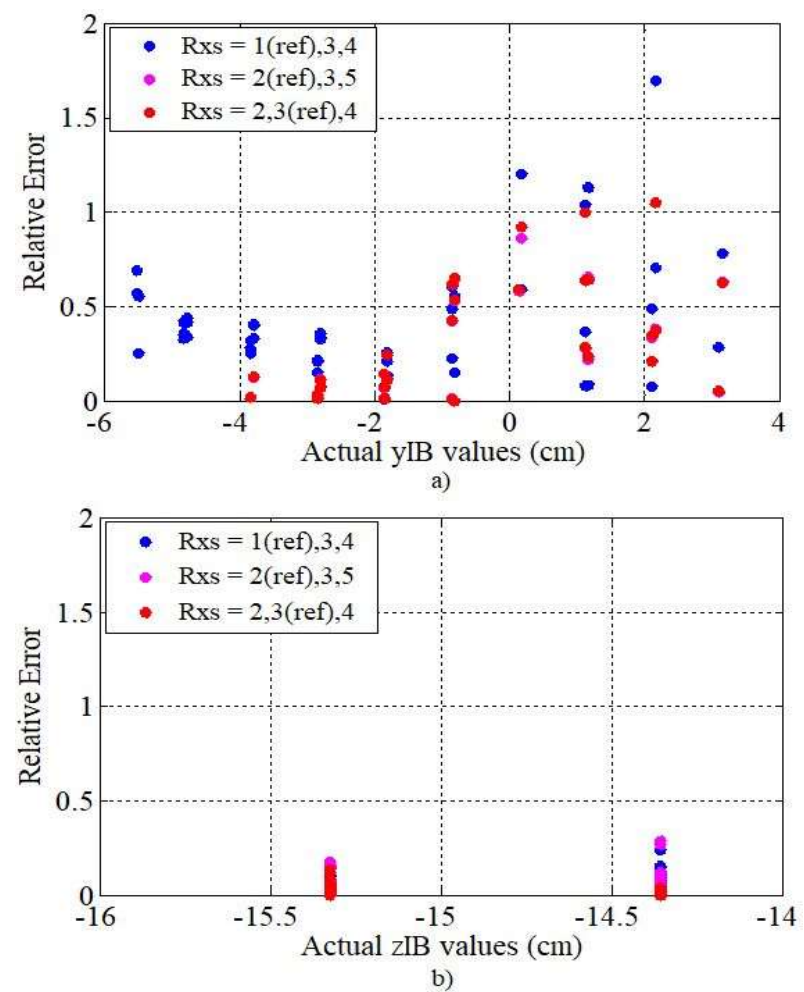

Figure 6. Relative errors on the estimation of $y_{I B} a$ ) and $z_{I B} \mathrm{~b}$ ).

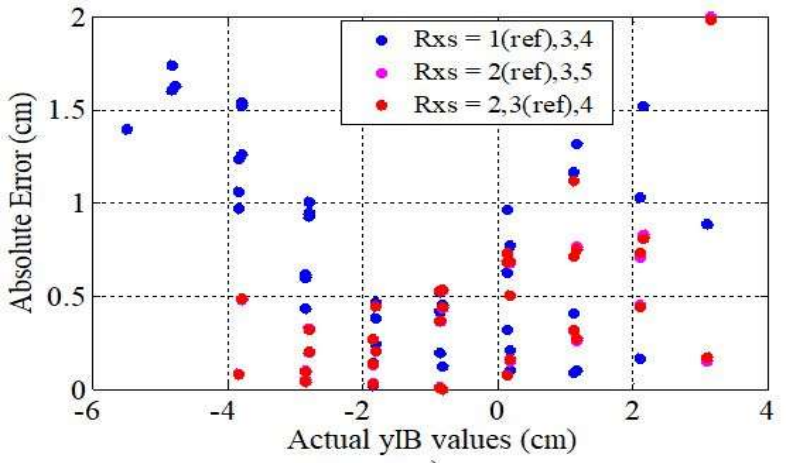

a)

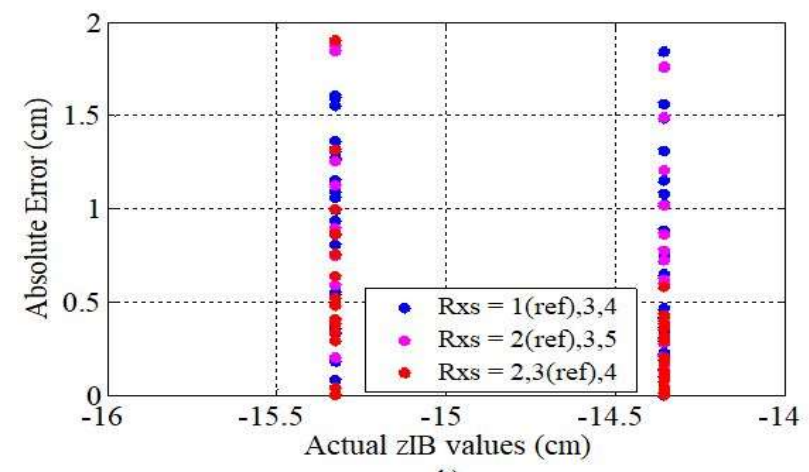

b)

Figure 7. Absolute errors on the estimation of $y_{I B}$ a) and $z_{I B}$ b).
One can observe that the absolute values of the error in both coordinates are very similar. Therefore, since $\left|z_{I B}\right|>\left|y_{I B}\right|$, the relative error calculated in Eqn. (11) and (12) results lower for the coordinate $z_{I B}$.

Furthermore, even in Figure 7 less variability in absolute error values can be observed for the combination of receivers 2 , 3 (reference), 4 in red color. Lastly, Figure 7 also shows that, in terms of absolute error, accuracy on average of $0.5 \mathrm{~cm}$ on the estimation of $y_{I B}$ and of $1 \mathrm{~cm}$ on the estimation of $z_{I B}$ can be achieved by employing RSS-based algorithm.

\section{CONCLUSIONS}

In this study the performance of RSS-based technique for 2D localization of in-body devices operating in UWB frequency band have been investigated. Analysis have been conducted through an experimental customized testbed employing a twolayer phantom model to emulate different human tissues. A first estimation of the in-body antenna coordinates by directly solving a system of two equations (2D case) in 2 unknowns has been obtained.

Results show that for distances up to $8 \mathrm{~cm}$ among transmitter and receiver a localization accuracy on average of $0.5-1 \mathrm{~cm}$ can be obtained with RSS-based approach. Furthermore, the location of the receivers used to estimate the in-body antenna coordinates plays a key role in achieving a precise localization.

In the future, minimization error algorithms will be implemented when more than three receivers (2D case) are used for localization, in order to improve the estimation of the inbody antenna coordinates. Additionally, ToA based-technique will also be investigated.

Further studies on the relationship between obtained results and the different location of the receivers combinations used should be carried out.

Finally, new experimental measurements with the presented custom-made setup will be conducted for $2 \mathrm{D}$ and $3 \mathrm{D}$ positioning. The main goal will be replicating the same measurements campaign presented here for all the edges of the multilayer phantom container in order to improve the localization accuracy of the in-body antenna.

\section{ACKNOWLEDGEMENT}

This work was supported by the European Union's H2020:MSCA:ITN program for the "Wireless In-body Environment Communication- WiBEC" project under the grant agreement no. 675353. This work was also funded by the Programa de Ayudas de Investigación y Desarrollo (PAID-0116) from Universitat Politècnica de València and by the Ministerio de Economía y Competitividad, Spain (TEC201460258-C2-1-R), by the European FEDER funds.

\section{REFERENCES}

C. Hu, M. Q. Meng, and M. Mandal, "Efficient Magnetic Localization and Orientation Technique for Capsule Endoscopy," in IEEE/RSJ International Conference on Intelligent Robots and Systems, 2005, 
[2] pp. 3365-3370

R. Kuth, J. Reinschke, and R. Rockelein, "Method for determining the position and orientation of an endoscopy capsule guided through an examination object by using a navigating magnetic field generated by means of a navigation device," US20070038063, 2007.

[3] G. Bao, L. Mi, and K. Pahlavan, "A Video Aided RF Localization Technique for the Wireless Capsule Endoscope (WCE) inside Small Intestine," in Proceedings of the 8th International Conference on Body Area Networks, 2013, no. February 2016, pp. 55-61.

[4] J. Lee, J. Oh, S. K. Shah, X. Yuan, and S. J. Tang, "Automatic classification of digestive organs in wireless capsule endoscopy videos," in Proceedings of the 2007 ACM symposium on Applied computing SAC 07, 2007, pp. 1041-1045.

[5] G. Bao and K. Pahlavan, "Motion estimation of the endoscopy capsule using region-based Kernel SVM classifier," in IEEE International Conference on Electro Information Technology, 2013.

[6] K. Pahlavan et al., "RF localization for wireless video capsule endoscopy," Int. J. Wirel. Inf. Networks, vol. 19, no. 4, pp. 326-340, 2012.

[7] M. Pourhomayoun, M. Fowler, and Z. Jin, "A novel method for medical implant in-body localization," in Proceedings of the Annual International Conference of the IEEE Engineering in Medicine and Biology Society, EMBS, 2012, pp. 5757-5760.

[8] U. I. Khan, K. Pahlavan, and S. Makarov, "Comparison of TOA and RSS based techniques for RF localization inside human tissue," in Proceedings of the Annual International Conference of the IEEE Engineering in Medicine and Biology Society, EMBS, 2011, pp. 5602-5607.

[9] A. R. Nafchi, S. T. Goh, and S. A. R. Zekavat, "Circular arrays and inertial measurement unit for DOA/TOA/TDOA-based endoscopy capsule localization: Performance and complexity investigation," IEEE Sens. J., vol. 14, no. 11, pp. 3791-3799, 2014.

[10] Y. Geng and K. Pahlavan, "On the accuracy of RF and image processing based hybrid localization for wireless capsule endoscopy," in 2015 IEEE Wireless Communications and Networking Conference (WCNC), 2015, no. Wenc, pp. 452-457.

[11] G. Bao, "On simultaneous localization and mapping inside the human body (body-slam)," Ph.D. dissertation, ECE, WPI, Worcester, MA, USA, 2014.

[12] A. S. Bjørnevik, "Localization and Tracking of Intestinal Paths for Wireless Capsule Endoscopy,” Master Dissertation, DET, Norwegian University of Science and Technology, 2015.

[13] M. R. Yuce, H. C. Keong, and M. S. Chae, "Wideband communication for implantable and wearable systems," IEEE Trans. Microw. Theory Tech., vol. 57, no. 10, pp. 2597-2604, 2009.

[14] M. Kanaan and M. Suveren, "In-body ranging with ultra-wideband signals: Techniques and modeling of the ranging error," Wirel. Commun. Mob. Comput., vol. 2017, 2017.

[15] Y. Ye, "Bounds on Rf Cooperative Localization for Video Capsule Endoscopy," Ph.D. dissertation, ECE, WPI, Worcester, MA, USA, 2013.

[16] S. Castello-Palacios, C. Garcia-Pardo, A. Fornes-Leal, N. Cardona, and A. Valles-Lluch, "Tailor-Made Tissue Phantoms Based on Acetonitrile Solutions for Microwave Applications up to $18 \mathrm{GHz}$," IEEE Trans. Microw. Theory Tech., vol. PP, no. 99, pp. 3987-3994, 2016.

[17] N. Cardona, S. Castello-Palacios, A. Fornes-Leal, C. Garcia-Pardo, and A. Valles-Lluch, "Synthetic Model of Biological Tissues for Evaluating the Wireless Transmission of Electromagnetic Waves," WO/2017/109252, 2017.

[18] S. Perez-Simbor, M. Barbi, C. Garcia-Pardo, S. Castello-Palacios, N. Cardona, "Initial UWB in-body channel characterization using a novel multilayer phantom measurement setup," in IEEE Wireless Communications and Networking Conference, WCNC, 2018, p. accepted for publication.

[19] C. Andreu, C. Garcia-Pardo, A. Fornes-Leal, M. Cabedo-Fabrés, and N. Cardona, "UWB In-Body Channel Performance by Using a Direct Antenna Designing Procedure," in 11th European Conference on Antennas and Propagation (EUCAP), 2017, p. 5.

[20] C. Tarín, P. Martí, L. Traver, N. Cardona, J. A. Díaz, and E. Antonino, "UWB channel measurements for hand-portable devices: A comparative study," in IEEE International Symposium on Personal,
Indoor and Mobile Radio Communications, PIMRC, 2007.

[21] R. Chandra, A. J. Johansson, and F. Tufvesson, "Localization of an RF source inside the Human body for Wireless Capsule Endoscopy," in Proceedings of the 8th International Conference on Body Area Networks, 2013, vol. 1, pp. 48-54. 